\title{
Conversion of Optic Neuritis to Relapsing Remitting Multiple Sclerosis: A Retrospective Comorbidity Cohort Study
}

\author{
Mesrure Koseoglu Mesude Tutuncu \\ Neurology Department/University of Health Sciences Istanbul Bakirköy Prof. Dr. Mazhar Osman Psychiatric Training and \\ Research Hospital, Istanbul, Turkey
}

\section{Keywords}

Multiple sclerosis · Optic neuritis · Central nervous sytem ·

Demyelination

\begin{abstract}
Objective: The aim of the study was to determine the risk of multiple sclerosis (MS) conversion after optic neuritis (ON) and to identify the predictive factors on conversion in Turkish patients. Methods: Patients whose first clinical attacks had been ON were included in the study. The primary end point was the diagnosis of clinical relapse-remitting MS. $R \boldsymbol{e}$ sult: Except for the bilateral involvement rate, the clinical and demographic characteristics of the cohort are similar to Western studies. Though one-third of the patients with ON had bilateral involvement, bilateral involvement reduces the risk of conversion. Also, active lesions at the initial cranial magnetic resonance imagination increase the conversion rate. Conclusion: This research confirms previous findings and contributes additional evidence that if the patients have unilateral involvement and active lesions, they should be closely monitored. Moreover, our research supports the hypothesis that risk factors may be affected by racial, environmental, and genetic factors.

(c) 2020 S. Karger AG, Basel
\end{abstract}

\section{Introduction}

Optic neuritis (ON) is an acute inflammatory demyelinating disease of the optic nerve [1]. The main clinical findings expected in demyelinating $\mathrm{ON}$ are visual disturbance, pain triggered by movement in the affected eye, impaired color vision, and decreased contrast sensitivity [2]. ON is usually observed in young adults. Symptoms mainly begin within hours or days and resolve within weeks. The recovery rate is usually high but very rarely permanent visual impairment occurs [3]. The most important issue to be considered, that is, ON may be the first sign of a demyelinating disease. $\mathrm{ON}$ is mainly associated with multiple sclerosis (MS). The first clinical finding in $20 \%$ of MS patients is ON and $90 \%$ of MS patients have electrophysiological evidence of ON [4]. On the other hand, the risk of conversion of ON to MS depends on the laboratory, clinical, and radiological parameters. The most significant factor that has the greatest effect on MS conversion is the appearance of lesions compatible with demyelination detected in the cranial or spinal magnetic resonance imagination (MRI). In a very comprehensive study conducted by the ON study group, it was reported that the risk of MS conversion was $72 \%$ in patients with 1 karger@karger.com www.karger.com/ene

(c) 2020 S. Karger AG, Basel

Karger"
Dr. Mesude Tutuncu

Department of Neurology, University of Health Sciences Istanbul Bakirköy Prof. Dr. Mazhar Osman Psychiatric Training and Research Hospital Bakirkoy, Istanbul 34147 (Turkey)

mesudeozerden@yahoo.com 
or more demyelinating lesions on MRI, and the risk was $25 \%$ in those without demyelinating lesion [5]. Other predictive risk factors identified in early studies are the presence of oligoclonal band (OCB) in the cerebrospinal fluid (CSF), gender, thinning of retinal nerve fiber layers, and the history of MS in the family members [6].

In 2015, McDonald's criteria were revised considering the importance of early treatment in MS. If the laboratory and radiological findings of the patients with ON meet the criteria for dissemination in time and space, MS can be diagnosed even in the first episode [7]. However, if these criteria are not met, selecting patients at high risk of conversion to MS is one of the main challenges that the neurologist faces. Although the conversion of $\mathrm{ON}$ to $\mathrm{MS}$ is one of the well-researched issues in the literature, the majority of the large-scale studies in this area include patients mostly in European and North American countries. So what we know about this conversion is largely based on studies conducted in populations of Western countries. Moreover, in recent studies, it has been argued that in Asian countries, the ON pattern differs from Western countries [8-10]. Interestingly, the onset of MS with ON was found to be higher in the Asian population. These rates are reported as 56\% in Taiwanese and 38\% in Chinese, while 20\% in Europeans [11]. However, most of the previous researches in Asian countries were cross-sectional, and there is a notable paucity of scientific literature describing the predictive factors of MS conversion. Very few studies have focused on this issue but found conflicting results from those in Western. Even if some of the differences observed between these results can be explained by patient selection or study end point; the impact of environmental, genetic, and racial features on the course is difficult to ignore. This study, therefore, was set out to assess the characteristics of patients with ON in Turkish patients and also to determine the risk factors for MS conversion.

\section{Materials and Method}

This retrospective study included patients admitted to the demyelinating diseases outpatient clinic at Bakirkoy Prof. Dr. Mazhar Osman Training and Research Hospital between 2008 and 2015. The records of all the patients who were admitted in this period were reviewed, and the patients with $\mathrm{ON}$ as a first clinical attack were included in the study. For the diagnosis of ON, patients had to show at least 2 of the following findings: pain with eye movements, decreased visual acuity, relative afferent pupillary defect, and prolonged P100 latency in visual evoked potentials.

The criteria to exclude patients are as follows:

- Patients with clinical evidence of a systemic disease other than MS
- Patients with repeating ON on the same or contralateral side

- Patients with proven conversion to non-MS demyelinating disease (e.g., neuromyelitis optica [NMO]) during the course (NMO antibody was measured during the course of all of the patients.)

Cranial MRIs of the patients during the attack of ON were examined. The number of white matter lesions with a diameter of at least $3 \mathrm{~mm}$ was calculated by an expert in neuroimaging. The presence of type 2 or 3 OCBs in CSF and/or IgG index higher than 0.7 were defined as positive CSF. Vitamin D levels during the first episode of $\mathrm{ON}$ were also examined. Serum $25(\mathrm{OH}) \mathrm{D}$ levels were evaluated using an electrochemiluminescence method. A normal level of vitamin $\mathrm{D}$ was defined as a $25(\mathrm{OH}) \mathrm{D}$ concentration $>30$ $\mathrm{ng} / \mathrm{mL}(75 \mathrm{nmol} / \mathrm{L})$, vitamin $\mathrm{D}$ insufficiency was defined as a $25(\mathrm{OH}) \mathrm{D}$ concentration between 20 and $30 \mathrm{ng} / \mathrm{mL}$ ( $50-75 \mathrm{nmol} / \mathrm{L}$ ) and vitamin $\mathrm{D}$ deficiency was defined as a $25(\mathrm{OH}) \mathrm{D}$ level $<20 \mathrm{ng} /$ $\mathrm{mL}(50 \mathrm{nmol} / \mathrm{L})$ according to previous studies [12]. Patients with a P 100 latency above $120 \mathrm{~mL} / \mathrm{s}$ or with a difference of $>10 \mathrm{~mL} / \mathrm{s}$ between both eyes were recorded as a positive visual evoked potential.

There were some certain drawbacks associated with creating a primary end point. One of these is that some ON patients were fulfilling the McDonald criteria for MS even in the first clinical attack, and according to McDonald's criteria, the presence of a new lesion was sufficient to diagnose RRMS for the others. However, it was not possible to take the presence of a new lesion as the primary end point for retrospective designed studies. It would cause bias. With all this in mind, to better understand the predictive factors in the Turkish population, the diagnosis of MS was based on Poser's clinical criteria similar to the study of the ON study group. Poser's criteria requires a second clinical attack, which lasted for at least $24 \mathrm{~h}$ and was attributable to central nervous system demyelination, consistent with neurological symptoms separated by at least 4 weeks from the first $\mathrm{ON}$ attack. In this article, the starting point of the study was the ON episode, and the primary end point is the second clinical episode. Disease-modifying therapy (DMD) exposure was defined as DMD usage of $>6$ months during the course.

In the first analyses, the clinical and demographic characteristics of the cohort were examined. In the second analysis, patients who convert to MS and patients who did not were compared in terms of clinical and demographic factors. In the third analysis, the factors affecting MS conversion were tried to be identified. Since some variables, such as vitamin D levels, were only reached in $30 \%$ of the patients, the values obtained from at least $60 \%$ of patients were added to regression and difference analyses in order not to reduce the statistical power. For patients who remained as clinically isolated syndrome without a second attack, the last visit dates were used as censor dates for analysis. The main variables included in the model were age (age at ON attack), gender, MRI findings, CSF findings (at any time), and DMD usage.

\section{Statistical Analyses}

All analyses were performed using SPSS software (version 20). $\chi^{2}$ test and, if necessary, Fischer's test were used to compare the ratios in categorical variables. The nonparametric Mann-Whitney $U$ test was used to compare the instruments of the 2 groups that were not normally distributed and the normal distributions were compared by using the Student's $t$ test. A $p$ value of $<0.05$ was considered statistically significant. The difference between the groups 
Table 1. Demographic, clinical, and radiological characteristics of the patients

\begin{tabular}{ll}
\hline Patients $(N: 104)$ & $\begin{array}{l}\text { Sociodemographic, clinical, and } \\
\text { radiological characteristics }\end{array}$ \\
\hline Female/male $(n)$ & $73 / 31$ \\
Age at ON attack & $30.48 \pm 8.68$ (min: 15-max: 51) \\
Vitamin D level at first attack, \% & 65.2 \\
$\leq 20$ & 17.4 \\
$20-30$ & 17.4 \\
$\geq 30$ & 21.3 \\
DMD usage, \% & 62.5 \\
Positive CSF, \% & 67.3 \\
Unilateral ON, \% & 32.7 \\
Bilateral ON, \% & 61.9 \\
Determination of prolongation in the latency of VEP, \% & 42.3 \\
Presence of $\geq 9$ periventricular lesions on cranial MRI, \% & 7.6 \\
Infratentorial involvement, \% & 34.6 \\
Presence of contrast-enhancing lesion, \% & \\
\hline
\end{tabular}

ON, optical neuritis; DMD, disease-modifying therapy; MRI, magnetic resonance imagination; CSF, cerebrospinal fluid; VEP, visual evoked potential.

was compared with the log-rank test by Kaplan-Meier analyses. Cox proportional hazard regression analysis was performed for the factors affecting the second clinical attack. Hazard ratios $>2$ and 95\% confidence intervals which do not include 1 were considered as significant.

\section{Results}

One hundred four patients met the inclusion criteria and were included in the study. Of the 104 patients, 31 were male and 73 were female. The mean age at the time of $\mathrm{ON}$ attack was $30.68 \pm 8.80$ years (min: 15.30 -max: 51.72 ). During the first episode of ON, $65.2 \%$ of patients had vitamin D deficiency and $17.4 \%$ of patients had insufficiency, and only $17.4 \%$ of patients had adequate vitamin $\mathrm{D}$ levels. Positive CSF was detected in $62.5 \%$ of the patients. Unilateral involvement was observed in $67.3 \%$ of the patients. The positive evoked potential was detected in $61.9 \%$ of the patients with ON. At the time of initial diagnosis, $34.6 \%$ of the patients had contrast-enhancing lesions on cranial MRI of whom $42.3 \%$ had 9 or more periventricular lesions. Infratentorial involvement was detected in $7.6 \%$ of the patients. During the course, $21.3 \%$ of the patients had DMD usage. All clinical, radiological, and sociodemographic characteristics of the patients are given in Table 1.

Eighty-three (79.9\%) of 104 patients had a second clinical attack compatible with demyelinating disease.
Table 2. Kaplan-Meier survival analysis according to demographic and clinical characteristics

\begin{tabular}{lll}
\hline & $\begin{array}{l}p \text { value } \\
(\text { log-rank })\end{array}$ & $\chi^{2}$ \\
\hline Positive CSF findings & 0.8 & 0.4 \\
Female/male & 0.14 & 2.1 \\
Presence of GD enhancing lesion & 0.05 & 4.9 \\
Unilateral/bilateral involvement & 0.03 & 4.9 \\
Periventricular lesions $\geq 9$ & 0.45 & 1.6 \\
DMD usage & 0.61 & 0.2 \\
Infratentorial lesion & 0.48 & 1.47 \\
\hline
\end{tabular}

CSF, cerebrospinal fluid; DMD, disease-modifying therapy.

No new episodes were observed in 21 patients. The patients had a second attack in a mean of 2.74 years. The mean follow-up period was $7.4 \pm 5.2$ years and the mean follow-up time of the non-converters was $3.28 \pm 1.04$ years.

There was no difference in terms of gender, positive CSF findings, and presence of periventricular lesions. However, those with an active lesion on the initial cranial MRI and those with unilateral onset were more likely to convert to MS (Table 2; Fig. 1). The effects of the clinical and radiological variables on the course were calculated by Cox proportional hazard regression analysis (Table 3). 
Fig. 1. Kaplan-Meier survival analysis according to bilateral/unilateral involvement at onset. ON, optical neuritis.

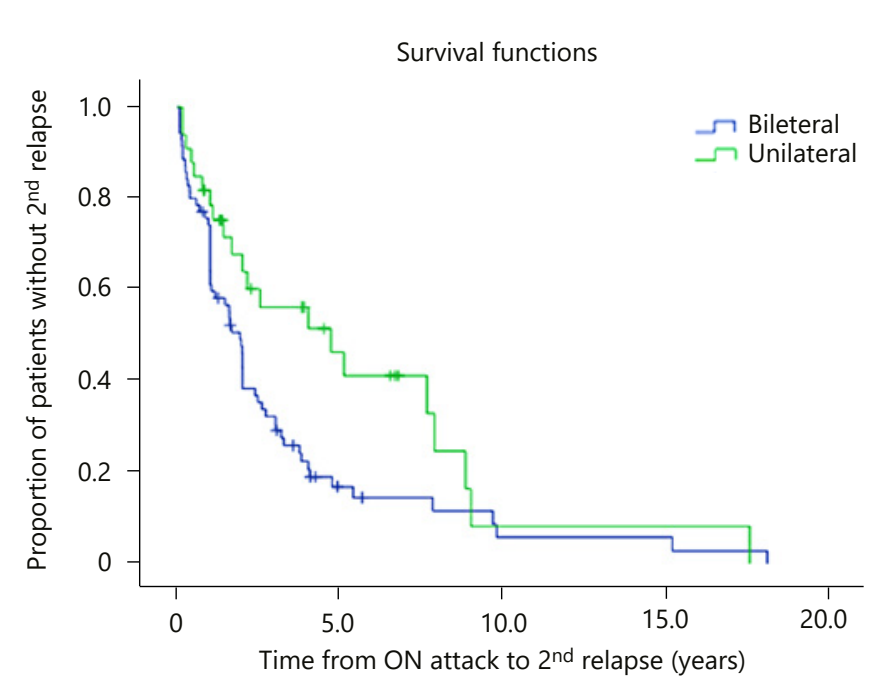

Table 3. Effects of the clinical and radiological variables on the course were calculated by Cox proportional hazard regression analysis

\begin{tabular}{llllll}
\hline & Sign $(p$ value $)$ & SE & Exp B & & \multicolumn{2}{c}{95 confidence interval } \\
\cline { 4 - 6 } & & & & lower limit & upper limit \\
\hline Age, years & 0.83 & 0.01 & 1.003 & 0.97 & 1.02 \\
Gender (F/M) & 0.09 & 0.27 & 0.63 & 0.37 & 1.08 \\
DMD usage $( \pm)$ & 0.39 & 0.29 & 0.77 & 0.43 & 1.39 \\
Contrast-enhancing lesion $( \pm)$ & 0.1 & 0.27 & 1.55 & 0.91 & 2.65 \\
Infratentorial lesion $( \pm)$ & 0.56 & 0.45 & 0.77 & 0.31 & 1.88 \\
Bilateral/unilateral ON & 0.11 & 0.28 & 2.09 & 1.18 & 3.68 \\
Positive CSF findings $( \pm)$ & 0.87 & 0.24 & 1.04 & 0.64 & 1.69 \\
\hline
\end{tabular}

CSF, cerebrospinal fluid; DMD, disease-modifying therapy; ON, optic neuritis.

\section{Discussion}

The relationship between ON and MS has long been known as ON may be the first clinical sign of MS. Considering the importance of early treatment in MS, it is important to predict the patients at high risk for developing MS. However, these risk factors may be affected by racial, environmental, and genetic factors, and there is a large gap in the literature in Asian and Latin countries. Therefore, in this study, we aimed to determine the characteristics of the patients with $\mathrm{ON}$ and to determine the risk factors for MS conversion in the Turkish population.

The first question in this study sought to determine the characteristics of the patients with ON. There was a female dominance in our cohort, supporting the usual fe- male preponderance. The mean age of the patients was similar to a cohort of the ON study group. Nearly half of the patients had $>9$ periventricular lesions on cranial MRI and nearly 3 of the 4 patients had at least 1 lesion on cranial MRI. In the ONTT trial, only $50 \%$ of the patients had 1 or more lesions in MRI, while in Asia, the rate was even lower $[5,8,10,13,14]$. It is difficult to explain this result, and it is possible that these results reflect a selection effect or ethnic factor taking part. In a study conducted in the same country, Durmus et al. [15] evaluated the patients with $\mathrm{ON}$. The author divided the patients into 2 groups as patients with $>1$ lesion and patients with $>3$ lesions, but no information was given about those without any lesions. A possible explanation for this might be that both of these 2 hospitals are a tertiary clinic for demyelinating 
diseases and patients with excessive lesion load may be referred to us.

More than half of the patients had positive CSF findings. Lumbar puncture was performed in only $31.5 \%$ of patients in the ONTT study but most of our patients had LP results. These results are in keeping with previous studies done by Frederiksen et al. [16], who reported that CSF abnormalities were present in $79 \%$ of idiopathic ON patients.

It is somewhat surprising that one-third of our patients had bilateral $\mathrm{ON}$ involvement at the onset. These results are consistent with previous studies done in Asian countries and suggest that this situation could be related to genetic and environmental factors [17]. Vitamin D deficiency was detected in most of the patients and this high prevalence seems to be related to the cultural characteristics of the geographical region we live in. In a previous study done in the same city, vitamin D levels of patients admitted to the outpatient clinic were found as follows: $43.1 \% 10 \mathrm{ng} / \mathrm{mL}, 31.9 \%$ between 10 and $20 \mathrm{ng} / \mathrm{mL}, 16.1 \%$ between 20 and $30 \mathrm{ng} /$ $\mathrm{mL}$, and only $8.9 \%>30 \mathrm{ng} / \mathrm{mL}$ [18]. The higher prevalence was associated with lifestyle patterns developed according to social and religious beliefs like clothing. Alagöl et al. [19] investigated the effect of dressing on vitamin $\mathrm{D}$ and found vitamin $\mathrm{D}$ deficiency in $60 \%$ of those dressed in Islamic style (the whole body closed or only hand and face open). Apart from all these, the associated vitamin D and demyelinating disease is now an accepted fact.

With respect to the second research question, $79.9 \%$ of the patients showed conversion to MS. This high rate can be explained by the fact that the majority of the patients in our study population had lesions on cranial MRI and the remaining patients had vitamin $\mathrm{D}$ deficiency except for $17 \%$. It is known that MRI has a conversion rate of $72 \%$ even in 1 lesion. As mentioned above, the studies evaluating this conversion rate varies in Asia. These results comply with the findings of Çinar et al. [20], in which the author reported the conversion rate of ON to MS as $85.7 \%$.

In our study, the presence of active lesion constituted one for the transformation of MS. The presence of an active lesion according to the criteria is already important in terms of dissemination criteria in space and time. The most interesting finding was the effect of bilateral involvement on the course. Twelve of 34 patients had returned to MS (64.1) and $87.12 \%$ of the patients with unilateral involvement had returned to MS. To the best of our knowledge, in the literature, there are a few studies suggesting that bilateral involvement reduces the risk. In addition, although not statistically significant ( $p: 0.09)$, in accordance with the literature, the female gender posed a risk for MS.

Conversion of Optic Neuritis to Relapsing

Remitting Multiple Sclerosis
One issue that may pose a bias for the study is how NMO patients are excluded. In our study, NMO and MOG antibodies were examined more than once in patients who did not convert to MS. Moreover, there were no lesions in the cranial MRI that would be compatible with NMO, including area postrema, periependymal regions, diencephalic structures, and corticospinal tract. But this distinction will always be the subject of controversy and cretaes a bias for all studies. Because $60 \%$ of patients with NMOSD accumulate asymptomatic white matter lesions and as many as $16 \%$ fulfill even the criteria of Barkhof [21]. Moreover, detection of brain MR white matter lesions compatible with MS does not exclude the diagnosis of NMOSD. Considering the OCB may be positive in NMOSD and MRI lesions fulfill both diagnostic criteria, this distinction does not seem possible with current markers.

Although the study has successfully demonstrated the risk factors that affect MS conversion, it has certain limitations in terms of retrospective nature and patient selection. The question raised by this study is will risk factors be affected by racial, environmental, and genetic factors? Although the relationship between ON and MS is well documented, it should be examined in each geographical area. More information on this issue would help us to establish a greater degree of accuracy on this matter. Considering the importance of early treatment for MS, prospective and larger studies are needed to determine risk factors for ON. This research confirms previous findings and contributes additional evidence that suggests bilateral involvement reduces the conversion risk, while active lesions at the initial cranial MRI increases.

\section{Acknowledgement}

We want to show our gratitude to the patients for their support in this study.

\section{Statement of Ethics}

This study was approved by the Medical Ethics Committee of the University of Health Sciences Istanbul Bakirköy Prof. Dr. Mazhar Osman Psychiatric Training and Research Hospital and was conducted ethically in accordance with Declaration of Helsinki.

\section{Conflict of Interest Statement}

The authors have no conflicts of interest to declare.

Eur Neurol 2020;83:287-292

DOI: $10.1159 / 000507547$ 


\section{Funding Sources}

No funding bodies were involved in the design, collection, analysis, interpretation, or writing of the manuscript. The views expressed are those of the authors.

\section{References}

1 Kale N. Optic neuritis as an early sign of multiple sclerosis. Eye Brain. 2016;8:195-202.

2 Shams PN, Plant GT. Optic neuritis: a review. Int MS J. 2009;16(3):82-9.

3 Beck RW, Cleary PA, Backlund JC. The course of visual recovery after optic neuritis. Experience of the Optic Neuritis Treatment Trial. Ophthalmology. 1994;101(11):1771-8.

4 Abou Zeid N, Zeid NA, Bhatti MT. Acute inflammatory demyelinating optic neuritis: evidence-based visual and neurological considerations. Neurologist. 2008;14(4):207-23.

5 Brodsky M, Nazarian S, Orengo-Nania S, Hutton GJ, Buckley EG, Wayne Massey E, et al. Multiple sclerosis risk after optic neuritis: final optic neuritis treatment trial follow-up. Arch Neurol. 2008;65(6):727-32.

6 Mamarabadi M, Razjouyan H, Mohammadi F, Moghaddasi M. Assessment of outcome predictors after first attack of optic neuritis. Can J Neurol Sci. 2011;38(6):887-95.

7 Thomson J, Banwell B, Barkoff F, Carroll W, Coetzee T, Comi G, et al. Diagnosis if multiple sclerosis: 2017 revisions of the Mc Donald criteria. Lancet Neurol. 2017;17(2):162-73.

8 Wakakura M, Minei-Higa R, Oono S, Matsui Y, Tabuchi A, Kani K, et al. Baseline features of idiopathic optic neuritis as determined by a multicenter treatment trial in Japan. Optic Neuritis Treatment Trial Multicenter Cooperative Research Group (ONMRG). Jpn J Ophthalmol. 1999;43(2):127-32.

\section{Author Contributions}

All authors have made a substantive intellectual contribution to the submitted manuscript.
9 Chuenkongkaew W, Chirapapaisan N. Optic neuritis: characteristics and visual outcome. J Med Assoc Thai. 2003;86(3):238-43.

10 Bee YS, Lin MC, Wang CC, Sheu SJ. Optic neuritis: clinical analysis of 27 cases. Kaohsiung J Med Sci. 2003;19(3):105-12.

11 Woung L-C, Chung HC, Jou J-R, Wang K-C, Peng P-H. A comparison of optic neuritis in Asian and in Western countries. Neuro-Ophthalmology. 2011;35(2):65-72.

12 Malabanan A, Veronikis IE, Holick MF. Redefining vitamin $\mathrm{D}$ insufficiency. Lancet. 1998;351(9105):805-6.

13 Chang YC, Wu WC, Tsai RK. Prognosis of Taiwanese patients with isolated optic neuritis after intravenous methylprednisolone pulse therapy. J Formos Med Assoc. 2007; 106(8):656-63.

14 Wang JC, Tow S, Aung T, Lim SA, Cullen JF. The presentation, aetiology, management and outcome of optic neuritis in an Asian population. Clin Experiment Ophthalmol. 2001;29(5):312-5.

15 Durmus H, Kürtüncü M, Tüzün E, Akalın B, Mutlu M, Gülflen A, et al. Optik nöritli hastalarda multipl skleroz geliflimi: öngörücü etmenlerin analizi. Turk Norol Derg. 2009;15: 119-23.
16 Frederiksen JL, Larsson HB, Olesen J. Correlation of magnetic resonance imaging and CSF findings in patients with acute monosymptomatic optic neuritis. Acta Neurol Scand. 1992;86(3):317-22.

17 Peng JT, Cong HR, Yan R, Kong XY. Jiang HQ, Wei WB, et al. Neurological outcome and predictive factors of idiopathic optic neuritis in China. J Neurol Sci. 2015;349(1-2): 94-8.

18 Sezgin G, Ozturk G, turkal R, Caykara B. Vitamin D levels of outpatients admitted to a University Hospital in the Marmara region of Turkey over 3 years. J Med Biochem. 2019; 38(2):181-7.

19 Alagöl F, Shihadeh Y, Boztepe H, Tanakol R, Yarman S, Azizlerli H, et al. Sunlight exposure and vitamin D deficiency in Turkish women. J Endocrinol Invest. 2000:23(3):173-7.

20 Çinar BP, Özakbas S. Prediction of conversion from clinically isolated syndrome to multiple sclerosis according to baseline characteristics: a prospective study. Arch Neuropsychiatry. 2018;55:15-21.

21 Wingerchuk DM, Banwell B, Bennett JL, Cabre P, Carroll W, Chitnis T, et al. International consensus diagnostic criteria for neuromyelitis optica spectrum disorders. Neurology. 2015;85(2):177-89. 